\title{
L'exception congréganiste en droit de la concurrence
}

Julien Couard

\section{CpenEdition}

\section{Journals}

Édition électronique

URL : http://journals.openedition.org/rdr/393

DOI : $10.4000 /$ rdr.393

ISSN : 2534-7462

\section{Éditeur}

Presses universitaires de Strasbourg

Édition imprimée

Date de publication : 24 avril 2018

Pagination : $55-86$

ISBN : 979-10-344-0017-1

ISSN : 2493-8637

\section{Référence électronique}

Julien Couard, «L'exception congréganiste en droit de la concurrence », Revue du droit des religions [En ligne], 5 | 2018, mis en ligne le 25 novembre 2019, consulté le 19 novembre 2020. URL : http:// journals.openedition.org/rdr/393 ; DOI : https://doi.org/10.4000/rdr.393

\section{(c) (7) (8)}

La revue du droit des religions est mise à disposition selon les termes de la Creative Commons Attribution - Pas d'Utilisation Commerciale 4.0 International - CC BY-NC 4.0. 


\section{L'EXCEPTION CONGRÉGANISTE ENDROIT DELACONCURRENCE}

\section{Julien COUARD}

Université de Toulon, Centre de droit et de politique comparés Jean-Claude Escarras

\section{RÉSUMÉ}

Depuis une dizaine d'années, diverses juridictions et autorités administratives ont eu à se prononcer sur des pratiques de congrégations posant question au regard du droit de la concurrence. Dans l'ensemble, les décisions prises vont dans le sens de la reconnaissance d'un régime d'exception essentiellement justifié par l'identité propre des communautés concernées, laquelle est garantie par la liberté religieuse. Dans la tradition ordo-libérale allemande, l'organisation du marché intègre ainsi une liberté fondamentale majeure. Cette articulation mérite d'être mise en lumière pour surmonter l'idée parfois soutenue d'un régime privilégié quand il n'est que dérogatoire à juste titre.

\section{Abstract}

Over the last decade, various courts and administrative authorities have ruled on the practices of congregations raising questions under competition law. Overall, the decisions are in line with the recognition of an exceptional regime essentially justified by the specific identity of these communities, which is guaranteed by religious freedom. In the German ordo-liberal tradition, the market organization incorporates a major fundamental freedom. This articulation deserves to be highlighted in order to overcome the idea of a privileged regime, when it is only rightly derogatory. 
Q ue penser d'une congrégation - entendue comme institution religieuse de vie en communauté - qui organise le monopole de la desserte maritime de l'île dont elle est propriétaire? Que penser d'une congrégation qui exerce une activité d'hébergement à des prix de loin inférieurs à ceux de tout hôtelier alentour? Que penser d'une maison d'édition appartenant à une congrégation qui s'oppose à toute commercialisation des textes pontificaux par un autre éditeur congréganiste francophone? Que penser encore d'une congrégation qui perçoit des subventions publiques pour la construction d'une salle de conférences ensuite louée à des clients pour un prix désarmant tout loueur professionnel de salles? Que penser enfin d'une congrégation vivant essentiellement de la fabrication artisanale d'hosties, qui perd peu à peu tous ses clients au profit de fabricants industriels?

C'est en ces termes concrets qu'émerge la problématique du rapport des entreprises congréganistes au marché et à la concurrence.

À l'évidence, dès lors qu'une communauté religieuse ${ }^{1}$ exerce une activité économique, elle évolue sur un marché et, à ce titre, se doit, a priori, de respecter les règles de droit de la concurrence. Toutefois, l'on perçoit bien que la dimension religieuse étant porteuse de comportements spécifiques liés à l'exercice d'une liberté fondamentale - la liberté de religion -, se pose la question de la combinaison a fortiori de cette liberté avec des règles normalement dictées par l'ordre public économique pour protéger la libre concurrence sur le marché ${ }^{2}$. Le libéralisme économique a, en effet, été réglementé afin de lutter contre les abus potentiels ou réels de liberté de commercer émanant d'acteurs dont l'intérêt propre s'éloigne parfois trop du juste comportement attendu sur le marché au nom du bien commun.

Or il ressort de cette conception d'une réglementation du marché apportant des restrictions légitimes au jeu de la concurrence ${ }^{3}$, qu'elle ne peut faire abstraction des libertés fondamentales ni considérer que la liberté de commerce les hiérarchise en partant du postulat qu'elle aurait une valeur normative supérieure aux autres ${ }^{4}$. Les droits et libertés fondamentaux apparaissent en

1. Au sens d'une congrégation de religieux ayant une vie en commun selon des principes d'ordre gouvernant tous les aspects de leur existence.

2. Rappr. Th. PEZ, «L'ordre public économique», N3C 2015, Le Conseil constitutionnel et l'entreprise, p. 44; M.-A. FRISON-ROCHE, «Les différentes natures de l'ordre public économique», Arch. phil. dr. 2015, p. 105; Ph. Malaurie, «La notion d'ordre public économique», Rev. conc. consom., $\mathrm{n}^{\circ} 83$, janv. 1995, p. 47.

3. Rappr. G. DECOCQ, «La réglementation est susceptible de restreindre le jeu de la concurrence de manière illégitime», RJ com. 2015, p. 57.

4. Pour un ex. d'application pratique, V. Cass. com., 8 juill. 2008, nº 07-16.761: Bull. civ. IV, $\mathrm{n}^{\circ} 143$. 
effet comme un ensemble indivisible et d'égale valeur normative, applicable à la vie économique comme à toute vie en société, au marché comme à tout rapport entre sujets de droit.

Le fait religieux, pris comme une réalité présente sur le marché, renvoie donc à la nécessité d'articuler la liberté religieuse avec les autres libertés fondamentales et avec la liberté de commerce. L'on observe d'ailleurs à cet égard que la liberté d'association et la liberté d'entreprendre, sous tous ses aspects économiques ${ }^{5}$, tiennent compte de la liberté religieuse. Ceci conduit à ce que, dans le monde des affaires, des communautés religieuses puissent s'associer, entreprendre, commercer et, plus largement contracter, dans le respect de leur identité religieuse ${ }^{6}$. En ce sens, les libertés économiques, tout en portant l'économie, portent également et permettent à l'économie de porter, la liberté religieuse.

Loin d'être évidente en France où l'économie est encore souvent perçue comme relevant d'un fonctionnement naturel échappant à l'homme ${ }^{7}$, cette conception est largement développée en Allemagne grâce à l'influence des ordo-libéraux de l'école de Fribourg-en-Brisgau ${ }^{8}$, eux-mêmes situés dans la mouvance néo-classique de l'école autrichienne dont les influences chrétiennes sont d'ailleurs nettement perceptibles ${ }^{9}$. Il en ressort l'importance centrale de la place de l'homme, ce qui fait de l'économie une question politique en ce qu'elle implique des choix humains fondamentaux. Les ordo-libéraux appréhendent

5. C'est-à-dire à la fois la liberté de commerce et d'industrie, qui n'est pas stricto sensu un droit fondamental mais un principe général du droit, et la liberté contractuelle.

6. J. Cound, L'entreprise congréganiste en droit des affaires, Paris, Defrénois, « Doctorat \& Notariat », 2009; comp. Mgr S. TOMASI (observateur permanent du Saint-Siège auprès des Nations unies et des institutions internationales à Genève), intervention lors de la VI ${ }^{\mathrm{e}}$ session du Conseil pour les droits de l'homme, Genève, 14 sept. 2007: «La profession d'une religion en public ou en privé est une liberté qui entre effectivement non seulement dans le domaine des droits civils et politiques, et qui est donc liée à la liberté de pensée, d'expression et de culte, mais aussi dans le domaine du droit économique [...]»: «Saint-Siège: la liberté religieuse, un "pont" entre tous les droits de l'homme», 20 sept. 2007 : https://fr.zenit.org/ articles/saint-siege-la-liberte-religieuse-un-pont-entre-tous-les-droits-de-l-homme/ [consulté le 9 janv. 2018]; rappr. V. Champeil-Desplats, «La liberté d'entreprendre au pays des droits fondamentaux», RDT 2007, p. 19.

7. H. Rabault, «La constitution économique de la France», RFD const. 2000, p. 707, spéc. p. $726-727$.

8. F. BILGER, La pensée économique libérale dans l'Allemagne contemporaine, Paris, LGDJ, 1964; «L'école de Fribourg, l'ordolibéralisme et l'économie sociale de marché», 8 avr. 2005: http://www.blogbilger.com/blogbilger/2005/04/lcole_de_fribou.html [consulté le 9 janv. 2018].

9. V. par ex. Th. E. Woods Jr., The Church and the Market: a Catholic Defense of the Free Economy, Lanham, Lexington Books, 2005, introd. p. 1, spéc. p. 7 et s.; chap. I, p. 13 et s., spéc. p. 19, p. 25 et s. 
en effet l'économie selon un ordre général de la concurrence visant à la fois la liberté économique et la prospérité, tenant compte par là des aspirations de l'homme à la liberté et au bien-être matériel ${ }^{10}$. Or ceci revient à façonner le système économique selon des valeurs, au premier rang desquelles figure la dignité humaine. Il est alors aisé d'apprécier la portée économique d'une telle notion. Le droit allemand traduit ceci dans l'idée de libre développement de la personnalité ${ }^{11}$ dont le sens économique profond s'exprime dans le droit à l'initiative économique et à la liberté professionnelle ${ }^{12}$. Ainsi conçue, l'économie politique va de pair avec une forte conception juridique de son organisation et de sa direction, formant de la sorte la base de l'économie sociale de marché ${ }^{13}$.

Au-delà de l'Allemagne, les institutions européennes naissantes d'aprèsguerre ont été marquées par la pensée qu'économie et État de droit se trouvaient liés. L'idée centrale de l'établissement d'une constitution économique et sociale au centre de laquelle se situe le principe de liberté a donc été transposée au niveau européen sous forme de conventions internationales appuyées par des déclarations de droits. Nul ne devait être juridiquement exclu de la vie économique, car cela aurait conduit à l'exclusion sociale et, à terme, à la remise en cause de la paix civile, donc à une dégradation des droits et de la dignité de la personne humaine. C'est dire que l'économie était alors envisagée comme soutien à l'ensemble des libertés fondamentales, ce qu'elle est devenue réellement au cours des décennies ${ }^{14}$. La liberté religieuse

10. Rappr. C. PRiETo, «L'entreprise et ses concurrents», in Entreprise et liberté, actes des journées nationales de l'association Henri Capitant, Montpellier, 2005, Paris, Dalloz, 2008, p. 43, spéc. p. 50-51.

11. Art. $2 \& 1$ de la Loi fondamentale: «Chacun a droit au libre épanouissement de sa personnalité pourvu qu'il ne viole pas les droits d'autrui ni n'enfreigne l'ordre constitutionnel ou la loi morale.»

12. Art. 12 de la Loi fondamentale: «1) Tous les Allemands ont le droit de choisir librement leur profession, leur emploi et leur établissement de formation. L'exercice de la profession peut être réglementé par la loi ou en vertu d'une loi. 2) Nul ne peut être astreint à un travail déterminé sinon dans le cadre d'une obligation publique de prestation de services, traditionnelle, générale et égale pour tous. [...]».

13. H. J. THIEME, Soziale Markwirtschaft. Ordnungskonzeption und wirtschaftspolitische Gestal-

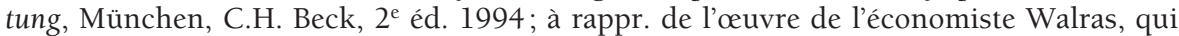
définit l'économie sociale comme «la partie de la science de la richesse sociale qui traite de la répartition de cette richesse entre les individus et l'État, et qui recourt au principe de la justice», autrement dit comme «la recherche des lois morales qui devraient présider à la répartition de la richesse sociale», cité in H. Guitton, Économie politique, Paris, Dalloz, $11^{\mathrm{e}}$ éd. 1976, p. 103, nº 120.

14. Rappr. par ex. A. BAilleux et N. TUlKENS, «Les droits fondamentaux dans l'ordre juridique de l'Union européenne», JDE 2016, p. 357; FranÇOIS, « Discours du Pape aux chefs d'État et de gouvernement de l'Union européenne à l'occasion du soixantième anniversaire des 
n’a donc pas été mise à l'écart ni de la pensée, ni de l'activité économiques; comme principe constituant de notre système de libertés fondamentales, elle a sa place dans les politiques ordonnatrices et régulatrices de l'économie ${ }^{15}$.

En conséquence, l'État se trouve obligé de concilier liberté religieuse et liberté d'entreprendre ${ }^{16}$, seules des exigences supérieures, comme le principe de laïcité par exemple ${ }^{17}$, pouvant restreindre ces libertés sans toutefois les supprimer ${ }^{18}$.

Traités de Rome sur la Communauté économique européenne et la Communauté européenne de l'énergie atomique, Rome, 24 mars 2017 », Osservatore Romano, éd. française, $\mathrm{n}^{\circ} 13,30$ mars 2017.

15. Ceci conduit à la gestion par les États du fait religieux sur le mode du pluralisme concurrentiel, dont le risque pour chaque religion repose dans le relativisme idéologique et s'exprime dans l'idée d'un "marché des croyances ou des religions » qui relève, selon nous, d'un point de vue juridique, davantage d'un abus de langage que d'une juste analyse du fait religieux. V. J. COUARD, L'entreprise congréganiste en droit des affaires, op. cit., $\mathrm{n}^{\circ} 588$; rappr. M. Ventura, La laïcità dell'Unione Europea. Diritti, mercato, religione, Torino, G. Giappichelli, 2001, p. 135 et s.; «Protectionnisme et libre-échangisme. La nouvelle gestion juridique de la religion en Europe», Conscience et liberté, n 64, 2003, p. 122; «La liberté de conscience dans la régulation publique de la religion. La compétition européenne», in J.-B. MARiE et P. Meyer-Bisch (éd.), Un noud de libertés. Les seuils de la liberté de conscience dans le domaine religieux, Bruxelles, Bruylant; Zürich, Schulthess, 2005, p. 133-157.

16. Comp. Mgr S. TOMASI, précit.: «Les pouvoirs publics [doivent] veiller à ce que la profession d'une religion ne limite pas l'accès à des droits civils ou à la participation politique et institutionnelle, ni ne soit utilisée pour nier aux individus ou aux communautés, l'accès aux droits économiques, sociaux et culturels.»

17. O. DORD, «L'affirmation du principe constitutionnel de laïcité», in L'architecture du droit. Mélanges en l'honneur de Michel Troper, Paris, Économica, 2006, p. 407.

18. C'est notamment ce qui ressort de l'affirmation par la CEDH que le principe de laïcité ne porte pas atteinte à la liberté religieuse: CEDH, $1^{\text {er }}$ juill. 1997, Kalaç c/ Turquie: Rec. 1997-IV, p. 1215, § 44, et mutatis mutandis, p. 1207, §§ 27-31; G. GonZALEZ (dir.), Laïcité, liberté de religion et Convention européenne des droits de l'homme, Bruxelles, Bruylant/Nemesis, 2006; A. GARAY, «La laïcité, principe érigé en valeur de la Convention européenne des droits de l'homme», D. 2006, chron., p. 103; rappr. J.-F. Flauss, «Les sources internationales du droit français des religions », LPA, 7 et 10 août 1992, p. 19 et p. 9; R. SĖve (dir.), Arch. phil. dr., t. 48, La laïcité, 2004. La CEDH admet en outre que des restrictions puissent être apportées à la liberté religieuse au nom de l'ordre public, notamment de la sécurité et de la santé publiques, V. CEDH, 13 déc. 2001, nº 45701/99, Église métropolitaine de Bessarabie et autres c/ Moldova, \& 113 (sécurité publique); comp. CEDH, 26 juill. 2007, n 10519/03, Barankevich c/ Russie: l'interdiction d'un culte protestant dans un jardin public au nom de la sécurité publique porte une atteinte injustifiée à l'art. 9 Conv. EDH; CEDH, Gde ch., 27 juin 2000, n² 2741/95, Cha'are Shalom Ve Tsedek c/ France, § 84: RTDH 2001, p. 191 (santé publique, à propos de l'abattage rituel casher; solution reprise en France, CAA Paris, 11 juill. 2007, n 06PA00631, Assoc. cultuelle israélite Cha'are Ha Shalom Ve Hatsedek: RLC 2008/14, nº 1008, obs. G. Clamour); rappr. E. TAWIL, Norme religieuse et droit français, Aix-en-Provence, PUAM, 2005, p. 243-244. 
Il ressort ainsi de cette combinaison, la soumission normale des congrégations au droit de la concurrence édicté par l'État (1) mais également la nécessité de tenir compte des spécificités induites par l'identité religieuse de ces acteurs sur le marché. Or ceci conduit parfois les juges et les autorités de concurrence à admettre que des atteintes à ce même droit de la concurrence par les congrégations puissent être justifiées (2).

\section{LA SOUMISSION DES CONGRÉGATIONS AU DROIT DE LA CONCURRENCE}

Par son objet même, le droit de la concurrence régit les rapports entre les personnes soumises à la loi de l'offre et de la demande de produits et services ${ }^{19}$. C'est dire qu'il ne s'attache qu'aux échanges commerciaux et aux activités économiques. Par conséquent, seule l'activité économique congréganiste relève des règles de concurrence, si bien que la notion de marché, lieu théorique et notion centrale de l'application du droit de la concurrence, est inapte à saisir le fait congréganiste dans son entièreté religieuse (1.1). Le saisissement ne s'opère que grâce à la notion d'entreprise, c'est-à-dire dès lors que les congrégations ont une activité économique au sens du droit de la concurrence (1.2).

\subsection{LA RELIGION N'EST PAS UN MARCHÉ AU SENS DU DROIT DE LA CONCURRENCE}

Si la liberté religieuse, comme constitutive de notre système de libertés fondamentales, a bien sa place dans les politiques ordonnatrices et régulatrices de l'économie, l'un des effets, que l'on peut qualifier de pervers, de l'ordo-libéralisme est de conduire à la gestion par les États du fait religieux sur le mode du pluralisme concurrentiel, traduit par la considération d'un "marché des croyances ou des religions ${ }^{20}$ ». Or il y a là un abus de langage faussant la juste analyse du fait religieux. En effet, toute activité religieuse

19. Rappr. M.-A. Frison-Roche, «Le modèle du marché», Arch. phil. dr., t. 40, 1996, p. 287.

20. Sur le constat et l'analyse de cette manière d'envisager la religion à l'échelle de l'Union européenne, V. M. Ventura, La laïcità dell'Unione Europea..., op. cit., p. 135 et s.; «Protectionnisme et libre-échangisme. La nouvelle gestion juridique de la religion en Europe», art. cit., p. 122 et s.; B. ChÉLini-Pont, «Religion in the Public Sphere: Challenges and Opportunities », Brigham Young University Law Review, n 3, 2005, p. 623 : l'auteur montre en quoi l'État organise l'entrée des religions dans la sphère publique par la normalisation des cultes au regard des libertés publiques et les met sur un pied d'égalité pour éviter toute discrimination. C'est dire, selon nous, qu'il contrôle l'accès au marché et assure le libre choix du fidèle selon un modèle de marché concurrentiel; dans le même ordre d'idée, M. Montalvo, « Religión y democracia de mercado », Anales de la cátedra Francisco Suárez, 
n'étant pas économique, la notion de marché ne fournit pas une clé de lecture efficace à l'application du droit de la concurrence au fait religieux en général et au fait congréganiste en particulier. Juridiquement, la religion n'est pas un produit concurrentiel ni d'ailleurs de consommation.

\begin{abstract}
Il faut alors distinguer nettement la mise sur le marché de biens et services issus d'institutions congréganistes qui assument leur identité propre jusque dans la sphère commerciale, d'un hypothétique «marché des croyances» dont nombre d'auteurs, juristes ou économistes, affirment l'existence ${ }^{21}$. Les religions elles-mêmes affirment d'ailleurs que le marché ne peut expliquer et régir toutes les relations sociales ${ }^{22}$.
\end{abstract}

$\mathrm{n}^{\circ}$ 41, Religión y democracia, 2007, p. 147; L. Mayali (dir.), Le façonnage juridique du marché des religions aux États-Unis, Paris, Mille et une nuits, 2002.

21. En ce sens notamment, Ph. Simonnot, Les Papes, l'Église et l'argent. Histoire économique du christianisme des origines à nos jours, Paris, Bayard, 2005, spéc. p. 19 et s.; Le Marché de Dieu, Paris, Denoël, 2008; «La "part bénite" », Sociétal, n 57, Économie et religion, 2007, p. 70 et s.; Ch. DE Laubier, «Pour un libre marché des cultes», Le Monde, 2 déc. 2005: l'auteur propose la création par l'État d'une «autorité de régulation des religions (ARR)» et un dégroupage des lieux de culte catholiques (comme France Telecom pour l'utilisation du réseau téléphonique par des opérateurs concurrents) pour les affecter aux autres cultes chrétiens, soit une mise sous tutelle de la religion au prétexte d'un assainissement de la pratique religieuse par la mise en ouvre des règles de concurrence, au plus grand mépris de la séparation des Églises et de l'État, et du principe de laïcité; G. GonzALEZ, «Religions et marchands, marchands de religion. Arbitrages à la lumière de la Convention européenne des droits de l'homme», in Mélanges Yves Serra, Paris, Dalloz, 2006, p. 217 : le titre est évocateur et l'auteur traite de la liberté pour «l'homme du $\mathrm{xxI}^{\mathrm{e}}$ siècle» de trouver «le produit spirituel qui lui sied»; sur l'utilisation du concept de «marché des religions», même dans la doctrine du droit civil ecclésiastique, V. A. Guazzarotti, "L'accesso al "mercato religioso" in Italia. Note critiche sul finanziamento delle confessioni religiose", Dir. eccl. 1997, I, p. 116. L'idée d'une mise en concurrence des religions n'est pas nouvelle puisqu'elle avait été avancée dès le XIX ${ }^{e}$ siècle, notamment par Benjamin Constant, V. B. Constant, «Principes de politique», in Euvres, Paris, Gallimard «Bibl. de la Pléiade», 1957, p. 1227-1228. En outre, dès le XvıI' ${ }^{e}$ siècle, Adam Smith, dans ses Recherches sur la nature et les causes de la richesse des nations (1776), analysait l'Église sous l'angle économique, en prenant l'économie, dans une approche sociologique voire anthropologique, comme une méthodologie applicable à l'ensemble des rapports humains.

22. Concernant, par ex., la position de l'Église catholique, V. JEAn-Paul II, encycl. Centesimus annus, $\mathrm{n}^{\circ}$ 39, AAS 1991, p. 843: «La liberté économique n'est qu'un élément de la liberté humaine. Quand elle se rend autonome, quand l'homme est considéré plus comme un producteur ou un consommateur de biens que comme un sujet qui produit et consomme pour vivre, alors elle perd sa juste relation avec la personne humaine et finit par l'aliéner et par l'opprimer », également n 34 et 40 ; Conseil Pontifical Justice Et Paix, Compendium de la doctrine sociale de l'Église, Libr. Ed. Vaticana, 2005, n 349: «On ne peut souscrire à l'idée de pouvoir confier au seul marché la fourniture de toutes les catégories de biens, car une telle idée est basée sur une vision réductrice de la personne et de la société. Face au risque concret d'une "idolâtrie" du marché, la doctrine sociale de l'Église en souligne les limites, que l'on peut facilement relever dans l'incapacité constatée où il se trouve de satisfaire les exigences humaines importantes pour lesquelles il faut "des biens qui, 
Certes, des économistes ont pu montrer que le choix des individus pour adhérer à une religion pouvait s'analyser de la même façon qu'un achat, avec une part de rationnel et d'irrationnel, un coût plus ou moins élevé en fonction du degré d'exigence dans la foi contre l'obtention du salut ${ }^{23}$. Séduisante de prime abord, cette analyse trouve cependant rapidement ses limites. En effet, l'économie du salut n'est pas celle du marché24. Elle repose sur la gratuité. La valeur est à distinguer du prix et les valeurs n'y ont pas de prix dépendant de l'offre et de la demande ${ }^{25}$.

Du strict point de vue du droit français et européen, il est vrai que les convictions religieuses se valent et que le choix des individus s'exerce en principe librement. C'est pourquoi certains ont affirmé que cela induisait une concurrence entre religions pour attirer de nouveaux fidèles et conquérir des "parts de marché ${ }^{26} »$. Le langage et la rhétorique ont leurs facilités. La force du droit consiste à les ignorer pour ne pas tomber dans les travers d'une fausse apparence.

par leur nature, ne sont et ne peuvent être de simples marchandises", biens non négociables selon la règle de "l'échange des équivalents" et la logique du contrat, typiques du marché. ", également n 356; BENOÎT XVI, entretien avec le père E. von Gemmingen, responsable de la rédaction allemande de Radio Vatican, Castelgandolfo, 16 août 2005: «Et voilà alors le "marché des religions" qui d'une certaine manière lui aussi offre la religion comme une marchandise et qui donc la dégrade, certainement. » Certaines églises protestantes américaines entrent au contraire, dans cette logique de consumérisme et, par ex., ouvrent leurs locaux en fonction de leur rentabilité c'est-à-dire de leur taux de remplissage. Ainsi, décident-elles parfois de fermer pour Noël, préférant donner à leurs fidèles un DVD à regarder en famille en remplacement de la célébration. D’autres retransmettent la célébration sur l'internet pour éviter d'avoir à mettre en place une logistique trop importante (mobilisation de bénévoles pour le parking, le gardiennage des enfants, la librairie, la buvette...): V. Le Figaro, 24-25 déc. 2005.

23. Ph. Simonnot, op. cit. et art. cit.; M. McConnell and R. Posner, «An Economic Approach to Issues of Religious Freedom», University of Chicago Law Review, 56, 1989, p. 1; L. R. IAnnaccone, "The Consequences of Religious Market Structure», Rationality and Society, 1991, III-2, p. 156; «Religious Markets and the Economics of Religion», Social Compass, 1992, 1, p. 123; R. StARK and J. C. MCCANN, «Market Structure and Catholic Commitment Exploring the New Paradigm», Journal for the Scientific Study of Religion, 1993, p. 111; L. R. IAnNaccone, R. Finke and R. Stark, «Deregulating Religion: the Economics of Church and State », Economic Inquiry, 1997, p. 350; S. CREACO, « Towards an Economic Analysis of Religion», Il dir. eccl., 1998, I, p. 635.

24. Comp. E. Lasida, Figures économiques de la transcendance. Étude sur la logique du sacré et la logique du marché, thèse, Paris, Institut catholique, 1999; "Économie et religion: un dialogue à construire», Sociétal, n 57, Économie et religion, 2007, p. 74.

25. A. Michel, Capitalisme contre capitalisme, Paris, Seuil, 1991, p. 120-122: l'auteur compare les modèles capitalistes rhénan et américain et affirme que «les religions fonctionnent essentiellement dans le modèle rhénan, comme des institutions non marchandes [...]. Aux États-Unis, on peut considérer, semble-t-il, que les religions, en nombre croissant, sont de plus en plus gérées comme des biens mixtes, avec des méthodes de médiatisation publicitaire et de marketing de plus en plus sophistiquées».

26. M. Ventura, op. cit. et art. cit. 
Ainsi, le droit de la concurrence comme la jurisprudence en la matière n'envisagent pas de «marché des croyances», car celles-ci ne relèvent pas par essence de comportements économiques. Les croyances ont certes des manifestations extérieures, mais elles touchent avant tout au for interne. Avant d'entrer dans la logique de l'échange, elles s'attachent à ce qui est dû à l'homme en tant qu'homme, du fait de sa dignité. Elles relèvent, par conséquent, du domaine des libertés fondamentales et des droits de l'homme, non des règles sur les biens et services. C'est dire que le juge ni les autorités de concurrence n'ont pas compétence pour déterminer, à l'aune du droit de la concurrence, si l'État organise une situation oligopolistique dans les départements d'Alsace-Moselle par le maintien du concordat, si la seule présence d'une église dans un village est le signe d'un monopole de l'Église catholique, ou si l'aménagement coordonné des horaires de messes dans un canton est constitutif d'une entente. En d'autres termes, l'affirmation par un auteur selon laquelle la religion est une entreprise spécialisée dans la production de croyances est juridiquement fausse ${ }^{27}$.

En revanche, dès lors qu'une institution religieuse développe une activité économique, celle-ci tombe sous la coupe du droit de la concurrence au même titre que toute autre entreprise. C'est alors cette notion d'entreprise qui permet d'appréhender le fait religieux.

\subsection{LES CONGRÉGATIONS AYANT UNE ACTIVITÉ ÉCONOMIQUE SONT DES ENTREPRISES AU SENS DU DROIT DE LA CONCURRENCE}

L'entreprise est la notion-clé du droit de la concurrence, car elle permet aux règles juridiques d'approcher au plus près la réalité de toute activité économique. Le droit de la concurrence la définit d'ailleurs comme toute entité exerçant une activité économique c'est-à-dire offrant, dans un but lucratif ou non, des biens et services sur un marché donné, quels que soient son statut juridique et son mode de financement ${ }^{28}$.

27. A. Weber, De l'économie du Salut. Étude sur le dogme dans ses rapports avec la morale, thèse, Strasbourg, 1863.

28. T. Fouquet, V. Giacobbo-Peyronnel, C. Remy et J. Sladic, «Les règles de concurrence applicables aux entreprises», JDE 2017, p. 55; rappr. CJCE, 10 janv. 2006, aff. C-222/04, Cassa di Risparmio di Firenze et autres: LPA, 17-18 mai 2007, p. 4, note L. ArCELIN; CJCE,

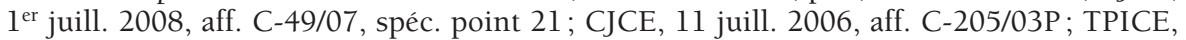
12 déc. 2006, aff. T-155/04. En droit français de la concurrence, il semble cependant que la jurisprudence nuance la définition selon les dispositions du livre IV du Code de commerce pour établir que certaine personnes morales exerçant une activité économique à but non lucratif ne soit pas toujours considérées comme des entreprises, V. Cons. constit., 
C'est dire qu'aucune entité congréganiste exerçant une activité économique ne devrait échapper à la qualification d'entreprise. En son temps, la Cour de justice des Communautés européennes a ainsi pu énoncer que «[...] la participation à une communauté fondée sur une religion [...] ne relève du champ d'application du droit communautaire que dans la mesure où elle peut être considérée comme une activité économique au sens de l'article 2 du traité ${ }^{29} »$. Les entreprises congréganistes peuvent, par conséquent, bénéficier des mesures du droit européen ou se les voir opposer. À leur avantage, elles peuvent, par exemple, profiter des libertés d'établissement ${ }^{30}$ et de circulation des personnes ${ }^{31}$ et des capitaux ${ }^{32}$. À leurs frais, elles ont notamment été tenues de respecter des quotas laitiers ${ }^{33}$.

Au niveau national, la question de la soumission de ces entreprises au droit de la concurrence, notamment lorsqu'elles revêtent la forme d'une congrégation légalement reconnue, a donné lieu à quelques tâtonnements jurisprudentiels. Ainsi, une cour d'appel, confirmant un jugement, a pu déclarer qu'une congrégation étrangère dont un établissement se livre, en France, à une activité commerciale habituelle, échappe au droit de la

7 janv. 2016, $\mathrm{n}^{\circ}$ 2015-510 QPC: RLC, $\mathrm{n}^{\circ}$ 48, 2016, p. 18, note L. ARCELIN; Contrats, conc. consom., mars 2016, p. 39, note D. Bosco; Cass. com., 8 févr. 2017, n 15-15.005: Contrats, conc. consom, mai 2017, p. 38, note G. DECOCQ; juin 2017, p. 33, note D. Bosco.

29. CJCE, 5 oct. 1988, aff. 196/87, Steymann: Rec. CJCE 1988, p. 6159; D. 1989, somm. p. 228, note L. Cartou; JDI 1989, p. 419, note M.-Ch. Boutard-Labarde.

30. Même affaire: condamnation des Pays-Bas par la Cour pour le refus à un membre d'une communauté religieuse (non catholique), d'un titre de séjour nécessaire à l'accomplissement d'une activité économique. Les juges rapportent un intéressant parallèle fait par la Commission entre le cas d'espèce et celui d'un frère technicien chargé de l'entretien des cuves de bière dans un couvent trappiste: Rec. CJCE 1988, p. 6164.

31. Rappr. CJCE, 4 déc. 1974, aff. 41/74, van Duyn: Rec. CJCE 1974, p. 1337 : désapprobation par la Cour du refus à un membre de l'Église de scientologie du droit d'entrée au Royaume-Uni en raison de la libre circulation des personnes dans l'espace européen sauf pour des motifs d'ordre public (non établis en l'espèce).

32. Rappr. CJCE, 14 mars 2000, aff. C-54/99, Association Église de scientologie de Paris et Scientology International Reserves Trust: la décision ne fait à aucun moment référence au caractère religieux de l'association, signe de l'absence de toute dérogation pour celle-ci aux règles juridiques communautaires en matière de circulation des capitaux.

33. CJCE, 23 nov. 1995, aff. C-285/93, Dominikanerinnen-Kloster Altenhohenau: Rec. CJCE 1995, p. 4069 : «1. L'article 12 sous $\mathrm{h}$ ), du règlement $\mathrm{n}^{\circ} 857 / 84$ doit, compte tenu tout à la fois de ses termes, lus en combinaison avec le même article sous c), et de l'objectif du régime de prélèvement supplémentaire, qui exige que toutes les quantités produites entrant, d'une manière ou d'une autre, dans le circuit commercial et influençant ainsi l'offre et la demande soient prises en compte, être interprété en ce sens que la livraison de lait effectuée par une exploitation agricole aux élèves et pensionnaires d'une école contre paiement indirect du prix du lait inclus dans le prix de la pension doit être qualifiée de vente directe au sens de cette disposition, et non d'autoconsommation, même lorsque l'exploitation agricole, l'école et le pensionnat sont gérés par la même institution.» 
concurrence en raison de son seul caractère religieux qui, juridiquement, la distingue nettement d'une association tant par son but que par son mode de création et de fonctionnement ${ }^{34}$. Cette décision est surprenante à bien des égards. D'abord, les juges ne se sont pas préoccupés de distinguer, d'une part, la situation, en France, des congrégations reconnues de celles qui ne le sont pas $^{35}$, ni, d'autre part, l'existence légale de la congrégation en Espagne, lieu de sa fondation, de la reconnaissance légale de son établissement en France. Prise dans son ensemble, la communauté religieuse a été considérée comme une congrégation distincte, par principe, d'une association, alors même qu'en l'absence de reconnaissance légale, celle-là peut prendre la forme juridique de celle-ci. L'application de l'article 37 de l'ordonnance du $1^{\text {er }}$ décembre 1986 (art. L. 442-7 C. com. ${ }^{36}$ ) a donc été écartée, les magistrats faisant ainsi une interprétation très stricte et littérale de l'article en le restreignant aux seules associations, sans chercher à en percevoir le sens au regard d'éventuelles dispositions statutaires permettant à une congrégation personne morale à but non lucratif d'avoir une activité économique à finalité religieuse. Partant, il faut en conclure qu'une congrégation personne morale de droit étranger peut accomplir une activité économique en dehors du droit de la concurrence. Or pourquoi une congrégation échapperait-elle a priori et par principe à ce droit, alors même qu'elle a, de facto, une activité commerciale habituelle en France? Du point de vue de la forme juridique, cela revient à dire que certaines entreprises sont assujetties au droit de la concurrence en fonction de leur statut légal, ce que tendrait d'ailleurs à admettre parfois la Cour de cassation ${ }^{37}$. Néanmoins, c'est oublier que cet assujettissement se fonde prin-

34. CA Pau, 17 sept. 1994, JurisData n 1994-047451, Syndicat des hôteliers restaurateurs de Lourdes cl Congrégation des religieuses de l'Amour de Dieu.

35. En France, certaines communautés religieuses appelées congrégations disposent du statut de congrégation légalement reconnue (L. $1^{\text {er }}$ juill. 1901) tandis que d'autres sont de simples associations de fait ou déclarées. Lorsqu'elles sont catholiques, toutes disposent en revanche d'un statut canonique.

36. «Aucune association ou coopérative d'entreprise ou d'administration, ne peut, de façon habituelle, offrir des produits à la vente, les vendre ou fournir des services si ces activités ne sont pas prévues par ses statuts. "L'activité économique d'une association est définie par la jurisprudence comme toute activité ayant pour objet la production ou la distribution de biens ou de services, dès lors qu'elle n'est pas exercée pour les besoins personnels de l'association mais pour satisfaire les besoins des tiers exprimés sur un marché, V. P. MACQueron, «Association et activité professionnelle», in Mélanges Mercadal, Paris, F. Lefebvre, 2002, p. 355, spéc. p. 366-367; Mémento Lefebvre, Associations, Fondations, Congrégations 2008-2009, n 11310 et s.; adde, M.-A. Frison-Roche et M.-S. PAYET, Droit de la concurrence, Paris, Dalloz, 2006, $\mathrm{n}^{\circ} 83$ et s.

37. V. Cass. com., 8 févr. 2017, précit.; comp. CA Versailles, 16 déc. 2004 : Bull. inf. C. cass. $2005, n^{\circ} 980$, pour l'application des règles de concurrence à deux associations, sans même les qualifier d'entreprises et caractériser leur activité économique, dès lors que les 
cipalement sur une analyse économique du comportement de l'entreprise et non sur sa personnalité juridique avec laquelle il ne coïncide pas ${ }^{38}$.

Plus grave encore, la décision de la cour de Pau signifie ensuite qu'une congrégation française, reconnue ou non, ayant une activité commerciale habituelle n'est pas une entreprise au sens du droit de la concurrence. Ceci constitue un non-sens. Heureusement, cet arrêt, condamnable du point de vue du droit, est resté isolé.

En 2005, une position plus réaliste du Conseil de la concurrence est venue corroborer le principe dégagé en droit communautaire. Dans la fameuse affaire de la desserte de l'île Saint Honorat, le Conseil a, en effet, pu connaître d'un conflit opposant une congrégation à une société de transport maritime en affirmant clairement sa compétence et le caractère économique de l'activité de la congrégation ${ }^{39}$. C'était admettre de qualifier d'entreprises en droit français de la concurrence des entités religieuses offrant leurs produits et services sur le marché, indépendamment de leur forme juridique.

Ceci pose, en conséquence, le problème de la spécificité de l'offre congréganiste et du besoin d'affiner la notion d'entreprise. En effet, cette offre n'est pas toujours aisée à situer sur un marché, car elle n'est pas systématiquement concurrentielle en raison de la finalité religieuse de l'activité poursuivie, laquelle peut concerner des produits, un public, des prix voire des méthodes de publicité qui l'éloignent de toute concurrence ${ }^{40}$.

D'abord, la qualification d'entreprise peut s'en trouver remise en cause pour certaines activités et l'application à celles-ci du droit de la concurrence apparaît parfois inopportune. Quid, à ce propos, de certains services éducatifs, d'hébergement ou de soins médicaux (écoles, hôtelleries, maisons

agissements de l'une tendaient à tirer profit des actions de l'autre en faisant l'économie des investissements correspondants.

38. V. par ex., en ce sens, M.-A. Frison-Roche et M.-S. PAYet, op. cit., p. 43-45, n 42-43.

39. Cons. conc., déc. n 05-D-60, 8 nov. 2005, Soc. Trans Côte d'Azur; Contrats, conc. consom. 2005, $\mathrm{n}^{\circ}$ 204: «43. Le Conseil de la concurrence est [...] compétent pour apprécier si l'activité de transport de passagers mise en œuvre par la Congrégation cistercienne de l'Immaculée Conception, par l'intermédiaire de la société Planaria, pour desservir l'île Saint Honorat respecte le jeu de la concurrence. Il s'agit d'une activité commerciale de prestation de service, au sens de l'article L. 410-1 du Code du commerce [...]»; nous revenons sur cette affaire, infra 2.1.

40. Cette grille d'analyse dite des «4 $\mathrm{P} »$ est appliquée par l'administration fiscale afin de déterminer l'assujettissement d'une entreprise constituée en personne morale à but non lucratif aux impôts commerciaux (impôt sur les sociétés, taxe sur la valeur ajoutée et contribution économique territoriale): BOI-IS-CHAMP-10-50-10-20, n 520 et s., spéc. $n^{\circ} 570$ et $\mathrm{s}$. 
de retraite, cliniques) qui oscillent entre la charité, le commerce lucratif et parfois, la mission de service public ${ }^{41}$ ? Ces services représentent bien une offre marchande dans le sens où ils sont substituables, ont un coût, répondent à des besoins. Pourtant, ils ne sont pas soumis entièrement au droit de la concurrence. Échappent à celui-ci les entités qui remplissent une fonction exclusivement sociale, fondée sur le principe de la solidarité et dépourvue de tout but lucratif ${ }^{42}$. C'est ce que le législateur traduit par la notion d'utilité sociale ${ }^{43}$.

En outre, ces organismes contribuent souvent à l'accomplissement de missions de service public, ce qui indique leur absence de pleine participation

41. V. par ex. CAA Nantes (2 arrêts), 7 juill. 1993, JurisData n ${ }^{\text {os }} 1993-047704$ et 047706 , Communauté de la Miséricorde: dans ces espèces, les juges retiennent le caractère concurrentiel de l'activité pour imposer celle-ci; contra CAA Nantes, 12 déc. 2005, JurisData $\mathrm{n}^{\circ}$ 2005-293741, Assoc. Famille et Joie.

42. TPICE, 4 mars 2003, aff. T-319/99, Federación Nacional de Empresas de Instrumentación Científica, Médica, Técnica y Dental; V. également le communiqué de presse n 12/03 s'y rapportant; ceci concerne surtout les organismes gérant un système d'assurance sociale, les syndicats et les ordres professionnels, M.-A. Frison-Roche et M.-S. PAYET, op. cit., $\mathrm{n}^{\circ} 84$ et s.; mais plus largement sur le fond, à rappr. A. Michel, Capitalisme contre capitalisme, op. cit., p. 123, n 7-8, pour l'enseignement et la santé; adde Fr. Perroux, Le capitalisme, Paris, PUF, «Que sais-je?», 1948, p. 105 et s. : "Toute société capitaliste fonctionne régulièrement grâce à des secteurs sociaux qui ne sont ni imprégnés ni animés de l'esprit de gain et de la recherche du plus grand gain. Lorsque le haut fonctionnaire, le soldat, le magistrat, le prêtre, l'artiste, le savant sont dominés par cet esprit, la société croule, et toute forme d'économie est menacée. Les biens les plus précieux et les plus nobles dans la vie des hommes, l'honneur, la joie, l'affection, le respect d'autrui, ne doivent venir sur aucun marché; faute de quoi, un groupe social quelconque vacille sur ses bases.»; p. 129-130: «[...] tout le prix de la vie vient de choses sans prix. Dans la sphère de l'acte gratuit, du don de ce qu'il possède et de ce qu'il est, l'homme atteint à ce qui constitue sa plus incontestable dignité.»

43. BOI-IS-CHAMP-10-50-10-20, n 590: «Est d'utilité sociale l'activité qui tend à satisfaire un besoin qui n'est pas pris en compte par le marché ou qui l'est de façon peu satisfaisante»; L. n 2014-856, 31 juill. 2014, relative à l'économie sociale et solidaire, art. 2: «Sont considérées comme poursuivant une utilité sociale au sens de la présente loi les entreprises dont l'objet social satisfait à titre principal à l'une au moins des trois conditions suivantes: $1^{\circ}$ Elles ont pour objectif d'apporter, à travers leur activité, un soutien à des personnes en situation de fragilité soit du fait de leur situation économique ou sociale, soit du fait de leur situation personnelle et particulièrement de leur état de santé ou de leurs besoins en matière d'accompagnement social ou médico-social. Ces personnes peuvent être des salariés, des usagers, des clients, des membres ou des bénéficiaires de cette entreprise; $2^{\circ}$ Elles ont pour objectif de contribuer à la lutte contre les exclusions et les inégalités sanitaires, sociales, économiques et culturelles, à l'éducation à la citoyenneté, notamment par l'éducation populaire, à la préservation et au développement du lien social ou au maintien et au renforcement de la cohésion territoriale; $3^{\circ}$ Elles concourent au développement durable dans ses dimensions économique, sociale, environnementale et participative, à la transition énergétique ou à la solidarité internationale, sous réserve que leur activité soit liée à l'un des objectifs mentionnés aux $1^{\circ}$ et $2^{\circ}$.» 
au libre jeu de l'offre et de la demande ${ }^{44}$. Certes, la forme juridique de l'entreprise congréganiste ${ }^{45}$ ou la règle fiscale des $« 4 \mathrm{P}^{46}$ » peuvent être de précieux indices caractéristiques d'une offre commerciale en la matière, mais elles ne sont que partiellement révélatrices des réalités marchandes et ne suffisent pas à délimiter précisément l'état de l'offre, remettant en cause la bonne application, voire l'application tout court, du droit de la concurrence.

Ensuite, lors même que l'activité économique congréganiste relève indubitablement de la notion d'entreprise, sa spécificité peut, malgré tout, conduire à s'interroger sur l'efficience du droit de la concurrence. Il se peut en effet que l'application de celui-ci à l'entreprise congréganiste pose question compte tenu de la prise en compte de la finalité religieuse de l'activité économique exercée par une communauté. Or force est de constater que, dans certains cas, le juge et les autorités de concurrence ont à la fois caractérisé des atteintes au droit de la concurrence commises par des congrégations et reconnu en même temps que ces atteintes étaient justifiées.

\section{LES ATTEINTES JUSTIFIÉES DES CONGRÉGATIONS AU DROIT DE LA CONCURRENCE}

L'application du droit de la concurrence à l'activité économique congréganiste fait tout de suite apparaître entre l'une et l'autre un certain nombre de distorsions. Celles-ci naissent de ce que les communautés religieuses n'ont pas la culture de la concurrence ${ }^{47}$. Bien au contraire, leur culture profonde est celle de la charité et de l'entente plutôt que des rapports de force, ce qui se retrouve jusque dans leur conception de l'activité économique ${ }^{48}$.

44. Car les prix et les prestations sont imposés: c'est le cas par ex. des tarifs de la Sécurité sociale, des modalités de paiement des frais (dans l'enseignement par ex.), des exigences publicitaires (dans l'enseignement ou dans le secteur médical, V. Cass. $1^{\text {re }}$ civ., 5 juill. 2006, JurisData $\mathrm{n}^{\circ}$ 2006-034432: concurrence déloyale d'une clinique ayant fait de la publicité pour ses médecins, ce qui est interdit), des programmes de cours, du recrutement et du paiement des personnels, de l'insertion dans des plans de répartition géographique des services publics.

45. Société commerciale par ex.

46. Sur cette règle qui suppose une analyse de la concurrence sur le marché, V. J. Couard, op. cit., $\mathrm{n}^{\circ} 787$ et s., spéc. $\mathrm{n}^{\circ} 789$ et $\mathrm{s}$.

47. C. Prieto, «La culture européenne de concurrence», in L'identité de l'Europe = Die Identität Europas, Actes du colloque de Tübingen, Aix-en-Provence, PUAM, 2002, p. 257.

48. V. par ex., Instruction Inter Prceclara pour la mise en pratique de la Constitution Sponsa Christi, 23 nov. 1950, n 27: «Les Supérieurs ecclésiastiques et religieux doivent: [...] 3) organiser avec soin la coordination des ouvres et du travail des divers monastères de 
Il est alors compréhensible que certaines pratiques d'entreprises congréganistes soulèvent des questions au regard de la bonne application du droit interne et européen de la concurrence.

La fracture entre ce droit et le fait religieux apparaît lorsqu'en dépit de la volonté des congréganistes de respecter la réglementation, un effort supplémentaire les obligerait à renoncer à leur identité collective ${ }^{49}$. Or la prise en compte de la liberté religieuse en droit de la concurrence appelle à rechercher une juste combinaison entre l'efficience de la règle de droit et le maintien de la finalité propre de l'entreprise congréganiste. Dans cette optique cependant, seules peuvent échapper au régime général de la matière les pratiques objectivement justifiées au regard de l'analyse économique de la concurrence.

Pour résoudre ces difficultés, deux sortes de solutions peuvent être alternativement ou cumulativement fournies: soit la communauté religieuse abandonne ses pratiques et se conforme pleinement au droit applicable à toute entreprise sur le marché, soit le droit applicable apparaît en trop grande contradiction avec son identité propre, compromettant sa finalité religieuse, et c'est à la norme de s'adapter.

La première éventualité devrait alors s'imposer chaque fois que la finalité religieuse de l'entreprise n'est qu'un prétexte à l'accomplissement d'une activité économique hors du champ légal. Cette hypothèse qui conduit à la pleine application du droit de la concurrence étant sans spécificité, elle ne sera pas abordée ${ }^{50}$.

La seconde devrait être retenue chaque fois que les pratiques de l'entreprise congréganiste sont objectivement justifiées sur le marché par la sauvegarde réelle de l'identité religieuse de son institution d'appartenance, appelant ainsi la mise en œuvre de solutions dérogatoires.

telle sorte qu'ils s'entraident les uns les autres, se remplacent, se complètent et que soit écartée de très loin même toute forme de rivalité. »

49. En témoignent ces quelques extraits, applicables à des communautés françaises, d'une charte éthique établie par la congrégation des sœurs hospitalières de Sainte Élisabeth, au Luxembourg: chap. VI, « § 2) Nous sommes en concurrence avec d'autres institutions et prestataires de service. Nous posons les accents et priorités nécessaires afin d'être et de rester compétitifs. § 3) En tant qu'organisation nous sommes ouverts à des concepts et visions modernes. Nous en vérifions la possibilité de réalisation et, tout en les adaptant aux situations qui se présentent, procédons à leur mise en œuvre. [...] § 5) Rentabilité et humanité doivent se compléter. Dans ce rapport de forces l'humanité doit l'emporter sur la rentabilité. Dans les solutions qui résultent de telles situations conflictuelles l'homme en tant que personnalité à respecter doit être au premier plan de toute considération. »

50. Pour davantage de précisions à ce sujet, V. J. COUARD, op. cit., nº 599 et s. 
Ceci est particulièrement vrai en matière d'abus de position dominante (2.1) et d'entente (2.2) mais plus incertain quant à la perception d'aides d'État (2.3). Dans le premier et le dernier cas, la jurisprudence s'est prononcée.

\subsection{L'EXONÉRATION DE SANCTION POUR ABUS DE POSITION DOMINANTE}

L'abus de position dominante est une pratique anticoncurrentielle, prohibée en tant que telle aux termes de l'article L. 420-2 du Code de commerce. Traditionnellement, les exemptions en matière de pratiques anticoncurrentielles étaient considérées comme improbables voire impossibles en droit français, relevant davantage d'une erreur de plume que de la volonté du législateur de se démarquer du droit européen qui n'en prévoit pas ${ }^{51}$. Pourtant, la jurisprudence s'est engouffrée dans la brèche, notamment à propos d'une affaire mettant en cause une entreprise congréganiste ${ }^{52}$.

En l'espèce, une congrégation cistercienne, propriétaire d'une île en face de Cannes, avait obtenu de l'État la concession des pontons afin d'organiser le transport commercial de personnes en direction du site. Néanmoins, au fil du temps, un seul ponton demeura, occasionnant des heurts entre compagnies maritimes autorisées par la congrégation à apponter. Le souci de rentabilité des compagnies conduisait, en outre, à une surfréquentation de l'île, ce qui dégradait le site classé et nuisait à la vie monastique. Pour remédier à ces maux, la congrégation créa alors sa propre compagnie, non sans avoir essuyé le refus de ses concurrents de collaborer au sein d'un groupement d'intérêt économique. Ainsi dotée, elle réserva l'unique ponton à sa société avec l'assentiment de l'Administration ${ }^{53}$. Pas moins d'une dizaine de requêtes furent alors déposées devant le tribunal administratif de Nice et le Conseil de la concurrence. Les requérants, dont un seul se maintint par la suite, désiraient obtenir le rétablissement de la concurrence sur la desserte de l'île, et la réparation, par l'État et par l'entreprise congréganiste, de leur préjudice commercial.

51. V. M.-A. Frison-Roche et M.-S. PAYet, op. cit., p. $215, \mathrm{n}^{\circ} 242$.

52. Rappr. C. Prieto, «La généralisation de l'analyse fondée sur les effets (ententes et abus de position dominante)», LPA 29 nov. 2007, Actualité 2006 en droit de la concurrence, p. 8 et s., spéc. p. 22.

53. Celle-ci refusa le droit aux autres compagnies de débarquer leurs passagers et s'opposa à la mise en œuvre d'une action en contravention de grande voirie à l'encontre de la congrégation qui avait fait démolir, après autorisation du concédant, le ponton devenu dangereux. 
À ce stade, il ressort clairement des faits que l'organisation économique mise en place par la congrégation avait pour but de préserver son identité propre, laquelle allie indissociablement la vie monastique au site puisque des moines y sont présents depuis le $\mathrm{v}^{\mathrm{e}}$ siècle.

\subsubsection{LE CONTENTIEUX ADMINISTRATIF}

Dans un premier temps, le tribunal administratif de Nice fit droit aux demandes des requérants en précisant que la convention de concession des pontons avait seulement pour objet et pour effet l'organisation par la congrégation du transport des passagers et non de lui en conférer le monopole ${ }^{54}$. Les juges regardèrent donc l'acte administratif qui entérinait la situation de monopole par l'affirmation de l'organisation d'une mission d'intérêt général ayant pour but d'assurer la continuité territoriale permettant l'accès au domaine public maritime et à un site remarquable, comme une délégation de service public. Or, n'ayant pas respecté les règles de mise en concurrence alors prévues par la loi du 29 janvier 1993, l'acte, entaché d'illégalité, fut annulé. C'était l'échec de la volonté de l'Administration de couvrir l'abus de position dominante de son concessionnaire par les nécessités de l'intérêt général ${ }^{55}$. Toutefois, le tribunal admit que l'ordre public économique restait soumis à cet intérêt général, lié à la continuité territoriale. L'accès au domaine public maritime de l'île n'était donc pas incompatible avec sa protection, ce qui justifiait que la congrégation pût organiser le transport tout en le limitant. Les règles de concurrence n'étaient ainsi pas évincées, mais le juge indiquait une contrainte que le concessionnaire pouvait imposer aux compagnies desservantes. Appel fut néanmoins formé par l'État et par la congrégation dont le monopole était remis en cause.

54. TA Nice, 15 avr. 2003, Soc. Trans Côte d'Azur et autres c/ Préfet des Alpes maritimes: Annuaire Droit et religions 2005, p. 401 (extraits), note J. COUARD; DMF 2003, p. 1010, note L. Bordereaux et S. Segala.

55. Les conventions d'occupation du domaine public sont généralement conclues à des fins purement commerciales. Néanmoins, soumises au droit de la concurrence lors de leur mise en œuvre économique, elles échappent toujours à une mise en concurrence préalable. La gestion du domaine public a, en effet, toujours été considérée comme relevant des pouvoirs de police administrative dans le seul but de profiter au bien commun. À cet égard, l'on estimait normal que les conventions domaniales s'assortissent de conditions qui les faisaient échapper au droit commun dès lors qu'était en jeu l'ordre public. L'on a cependant assisté à une lente dérive, passant de la gestion du bien commun à la recherche de la meilleure utilisation du domaine public. Les prérogatives de police administrative ont alors servi à établir et protéger la position dominante de concessionnaires à l'égard de leurs concurrents privés; V. Y. GAUdemet, Traité de droit administratif, t. 2, Paris, LGDJ, $13^{e}$ éd. 2008, p. 216, n 407 et s.; CE, 29 janv. 1932, Soc. des autobus antibois: Lebon, p. 117; D. 1932, 3, 60. 
La cour administrative d'appel de Marseille, pour sa part, adopta un raisonnement différent ${ }^{56}$. Plus pragmatique, elle préféra ne pas retenir la délégation de service public, pour deux raisons. Tout d'abord, la cour affirme dans son arrêt l'impossibilité et l'inutilité d'assurer la continuité territoriale entre le continent et l'île dès lors que le domaine public maritime de la côte insulaire est impraticable en raison de sa topographie ${ }^{57}$. Ensuite, elle précise que le classement du site ne suffit pas «à faire regarder l'organisation de sa visite par le seul propriétaire comme un service public touristique». Elle affirme donc l'application de la concession, laquelle attribue à la congrégation un droit exclusif d'organisation de la desserte de l'île.

De ce droit exclusif, elle tire celui de transporter par bateau des passagers à destination de l'île et précise qu'il «ne constitue pas, dans les circonstances particulières de l'espèce, tenant au statut juridique de l'île, à sa configuration et à la volonté de son propriétaire privé de limiter l'afflux touristique en vue d'y préserver les conditions de la vie monastique, un abus de position dominante contraire aux règles de la concurrence issues de l'ordonnance du $1^{\text {er }}$ décembre $1986 »$.

C'est dire que la cour admet l'existence d'un monopole de droit au profit de la congrégation, fondé sur une exclusivité accordée par l'État. Parmi les motifs essentiels ayant conduit la cour à se déterminer ainsi, il faut relever celui de «la volonté» de la congrégation «de limiter l'afflux touristique en vue d'y préserver les conditions de la vie monastique». Bien que ne figurant pas expressément dans la concession, ce motif déterminant est donc extrait de l'intention des parties, notamment de celle de la congrégation, pour donner tout son sens à la convention. L'opposabilité aux tiers concurrents ne fait alors plus de doute et ces derniers voient l'ordre public économique prendre sa place dans un intérêt général qui le dépasse, justifiant du même coup la situation monopolistique.

Plus largement, la convention aurait néanmoins probablement gagné en clarté par l'insertion des motifs déterminants lors de sa rédaction ou lors d'une éventuelle modification postérieure ${ }^{58}$.

56. CAA Marseille, 29 mai 2006, n 03MA01196.

57. Limité aux terrains baignés par les plus hautes mers en l'absence de circonstances météorologiques exceptionnelles, le domaine public maritime de l'île n'est formé que de rochers et de rares bancs de sable où s'amoncellent des algues.

58. Il est admis, dans les objectifs concernant l'évolution du trafic, que la protection du site, la qualité des services et la vitesse commerciale liée aux conditions de navigation sont susceptibles d'entrer dans l'objet d'une concession d'exploitation des services de transport public 
Quoi qu'il en soit, le Conseil d'État n'a rien trouvé à redire sur l'arrêt de la cour administrative d'appel de Marseille, reconnaissant ainsi aussi bien l'absence d'un service public de desserte maritime de l'île que l'existence d'un droit exclusif pour la congrégation de choisir les compagnies chargées d'assurer cette desserte ${ }^{59}$.

Sur le fond, il semble évident qu'implicitement l'État et les collectivités publiques ont reconnu la nécessité de préserver non seulement le site, mais également le droit et l'identité propre de l'institution congréganiste ${ }^{60}$. Sans quoi, il n'y aurait eu ni concession d'un droit d'exclusivité, ni protection de ce droit par des actes administratifs postérieurs.

\subsubsection{LE CONTENTIEUX DE LA CONCURRENCE}

Sans se prononcer sur les questions de droit administratif, le Conseil de la concurrence ne donna pas d'autre solution que celle de la cour administrative de Marseille ${ }^{61}$. Mieux, il l'inspira, puisque sa décision précéda l'arrêt de la cour ${ }^{62}$. Dans cette décision, le Conseil, après avoir écarté le grief d'entente ${ }^{63}$ et défini le marché pertinent, constate la situation monopolistique de l'entreprise congréganiste. De ce point de vue, à la différence de ce qu'a pu retenir ensuite la cour administrative de Marseille, il caractérise l'abus de position dominante. Puis il distingue entre les domaines privé et public de l'île. Il entreprend alors l'examen de la justification objective de l'abus, seule

de voyageurs. Pour une application, V. Droit des services publics locaux, t. 1, n IV.350.2, Paris, le Moniteur, 2001, p. 3, $\mathrm{n}^{\circ}$ 6, «Programme prévisionnel à long terme».

59. CE, 27 oct. 2009, n 297960, Soc. Trans Côte d'Azur.

60. Il est possible de débattre sur la question de savoir s'il ne s'agit pas là d'une aide indirecte, en l'absence de possibilité de verser des aides directes comme des subventions à des associations ayant une activité économique, et peut-être plus encore à une congrégation en raison de l'article 2 de la loi du 9 déc. 1905 («La République ne reconnaît, ne salarie ni ne subventionne aucun culte»). Une exception méconnue doit être cependant mentionnée concernant les aides directes: celle du droit local corse qui admet le versement de subventions et donc d'aides directes aux associations ayant une activité économique: L. 2002-92, 22 janv. 2002, art. 11, 17 et 19.

61. Cons. conc., déc. n 05-D-60, 8 nov. 2005, précit.: Contrats, conc. consom., déc. 2005, p. 18, n 204, note M. Malaurie-Vignal; RDC 2006/2, chron., p. 348, obs. C. Prieto; Concurrences, $\mathrm{n}^{\circ}$ 1/2006, chron., p. 137, obs. A. WACHSMANn; RTD com. 2006, p. 582, spéc. p. 584, obs. E. Claudel.

62. Dans son arrêt, la cour de Marseille n'hésite pas à mentionner la décision du Conseil de la concurrence.

63. Le Conseil rejette l'argument invoquant l'existence d'une entente entre la congrégation et la société dont elle est l'unique associé, au motif de l'absence d'un degré suffisant d'autonomie. Ceci va dans le sens de la reconnaissance d'une seule et même entreprise comprenant la congrégation légalement reconnue et sa société commerciale. 
de nature à exonérer la congrégation des sanctions normalement applicables par suite de l'inobservation du droit de la concurrence ${ }^{64}$.

Sur le monopole du transport vers la partie privée de l'île, il admet notamment que «l'objectif de limitation du nombre de visiteurs poursuivi par la Congrégation est justifié par la nécessité de préserver, sur un site peu étendu, la tranquillité du lieu monastique [...]». L'indéfectible lien entre la sauvegarde du site et de la vie monastique est donc bien à l'origine d'une justification objective du monopole.

En revanche, le Conseil constate que ce monopole a été étendu à la partie publique de l'île, c'est-à-dire au bord de mer. Cependant, après une analyse économique d'éventuelles solutions de remplacement, il justifie le monopole par la nécessité objective d'assurer la maîtrise du flux des visiteurs en raison de la géographie particulière des lieux, du caractère privé de l'île et de la volonté de la congrégation propriétaire ${ }^{65}$. Ainsi, est-il admis que la congrégation a su rendre indissociables l'accueil de personnes sur l'île et le transport maritime ${ }^{66}$. Et le Conseil conclut en précisant qu'«aucune solution permettant d'atteindre les mêmes objectifs mais présentant moins de restrictions de concurrence, n’a pu être mise en évidence».

Quelques mois plus tard, la cour d'appel de Paris confirma en tous points le raisonnement du Conseil de la concurrence ${ }^{67}$. Cette solution cohérente a ensuite été entérinée sur un autre terrain, celui de l'article 544 du Code civil, puisque la Cour de cassation a admis que violait ce texte la cour d'appel qui, tout en niant l'atteinte au droit de propriété de la congrégation propriétaire de l'île, constatait que la plate-forme dépendant du domaine public sur laquelle étaient débarqués les visiteurs transportés par une compagnie maritime non agréée par cette congrégation étant trop petite pour les contenir, les touristes amenés pénétraient nécessairement sur la propriété privée ${ }^{68}$.

Ainsi, déroge aux règles normalement applicables du droit de la concurrence la congrégation qui organise à travers son entreprise le monopole de la desserte de son île dans le seul but ultime d'assurer à ses membres la quiétude

64. V. C. com., art. L. 420-2 et L. 420-4.

65. L'argument présentant le ponton comme une infrastructure essentielle est écarté, car il ne résulterait d'une telle reconnaissance aucun progrès économique; sur la théorie des infrastructures essentielles, rappr. Cass. com., 12 juill. 2005: BICC 629, n 2100, annoté.

66. En ce sens, C. PRIETO, RDC 2006/2, chron. précit.

67. CA Paris, 4 juill. 2006, JurisData ${ }^{\circ}$ 2006-310182.

68. Cass. $3^{\mathrm{e}}$ civ., $\mathrm{l}^{\mathrm{er}}$ juin 2011, $\mathrm{n}^{\circ}$ 10-17.781: Annuaire Droit et religions 2012-2013, p. 719, note J. CouARD; dans le même sens après renvoi, CA Aix-en-Provence, 6 mars 2012, RG n 11/11439: Bull. Aix, 2012-2, p. 101, obs. J. COUARD. 
propre à la vie monastique. Il y a bien là une reconnaissance de la finalité propre de l'entreprise, au point de justifier en droit une situation préalablement qualifiée d'abusive. À ce propos, un auteur en arrive à la conclusion que «la tranquillité des religieux est donc une valeur non économique prise en compte par le Conseil de la concurrence ${ }^{69} »$. Pour un autre, la décision révèle véritablement «les justifications objectives d'une entreprise de tendance» qui, en l'espèce, ont conduit le Conseil à «absoudre l'exclusivité ${ }^{70} »$. C'est dire que l'on voit poindre en doctrine l'idée que l'entreprise congréganiste puisse être catégorisée en droit de la concurrence ${ }^{71}$.

\subsection{L'EXONÉRATION DE SANCTION POUR ENTENTE}

Bien que n'ayant jusqu'à présent donné lieu à aucune décision de justice ou de l'Autorité de la concurrence, la question de l'exonération de sanction en matière d'entente semble déboucher sur une réponse identique à ce qui a pu être retenu en matière d'abus de position dominante si l'on veut bien considérer l'exemple, tiré de la pratique, des réseaux organisés d'entreprises congréganistes.

Ces réseaux naquirent du développement massif de l'activité économique congréganiste à partir des années $1950^{72}$. Pour les communautés urbaines, majoritairement féminines et vouées à la vie contemplative notamment, il ne fut pas question de rompre la règle de vie et la clôture pour fonder une boutique. Aussi se mirent conjointement en place, dès 1948-1949, l'association d'Aide au travail des cloîtres (ATC) et des fédérations de plusieurs monastères d'un même ordre, afin de sensibiliser les religieux aux pratiques commerciales et de gestion économique ${ }^{73}$. Sans disparaître, ces initiatives

69. M. Malaurie-Vignal, précit., p. 12.

70. C. PriETo, RDC 2006/2, chron. précit. : l'auteur pressent toute la richesse de cette décision pour la théorie de l'analyse économique en droit de la concurrence, à l'heure où des voix s'élèvent contre l'assimilation entre l'efficacité économique et la pure logique financière.

71. Rappr. J. COUARD, op. cit., $\mathrm{n}^{\circ} 636$ et s.

72. La Constitution apostolique Sponsa Christi de Pie XII en 1950 fut véritablement à l'origine de la naissance de points de vente et de réseaux de distribution dans un objectif de rentabilité. Celle-ci bouleversa la vision qu'avaient les monastères du monde économique et des formes modernes d'organisation du travail. La première évolution, qui eut lieu peu de temps avant cette réforme de Pie XII, avait seulement consisté à créer des magasins sur le lieu de la communauté lorsque les locaux, le nombre de membres et la production le permettaient. Toutefois, ceci ne concernait principalement que les monastères ruraux vivant selon la règle de Saint-Benoît.

73. Sour GARREt, Un survol de l'évolution économique des monastères de moniales durant la seconde moitié du XX siècle, suppl. à Les Amis des monastères, n 146, avr. 2006, p. 10; 
prirent de l'ampleur, et s'y ajoutèrent la Fondation des monastères en 1969, dont les fonds permirent notamment la modernisation des outils et des techniques de production par le financement de matériels et de formations, puis l'association Monastic en 1989 avec sa marque éponyme.

Toutes ces organisations qui structurent l'offre congréganiste sur le marché ont pour but de permettre aux institutions congréganistes de se procurer des revenus par leur activité économique dans le complet respect de leur identité religieuse. Elles sont constituées principalement en réseaux associatifs à partir desquels se sont développés des réseaux de sociétés commerciales plus ou moins autonomes ${ }^{74}$. Les plus importantes d'entre elles sont l'association d'Aide au travail des cloîtres et l'association Monastic dont la situation vaut d'être vue à la lumière des dispositions de l'article L. 420-2 du Code de commerce prohibant les ententes.

\subsubsection{L'ASSOCIATION D'AIDE AU TRAVAIL DES CLOITTRES}

Officiellement fondée en 1951, cette association a pour vocation d'aider les communautés à choisir un travail dans lequel elles puissent agir en harmonie avec leur règle de vie propre ${ }^{75}$. Cette aide se réalise par le conseil, mais également par la formation des religieux au métier qu'ils décident d'accomplir, par la fourniture des matériels nécessaires à la réalisation des tâches, et par la commercialisation des produits ainsi conçus. L'objet de cette association ne se limite donc pas à une aide qui laisserait la communauté libre de la gestion de ses affaires économiques, mais consiste en un véritable partenariat liant l'association aux religieux qui y font appel. À cette fin, et au-delà de tout l'aspect d'assistance apportée par l'association, a été créée en 1956 une SARL dont le but est de vendre les produits monastiques et de faire des bénéfices en vue de s'autofinancer ${ }^{76}$. Cette filiale, connue sous le nom

l'expérience, concluante, déboucha sur la création de ce qui deviendra rapidement le Service des moniales (SDM) réunissant, autour de toutes sortes de thèmes parmi lesquels l'économie, l'ensemble des communautés de moniales contemplatives. Le mouvement fut sensiblement identique concernant les communautés masculines.

74. Les réseaux associatifs sont multiples et comptent de nombreuses entités à l'importance et à la notoriété variables: associations d'amis d'un monastère, fédérations de monastères d'un même ordre, alliances inter monastères, conférences de supérieurs majeurs. Certaines associations ne sont pas déclarées ou ne constituent que des groupes informels, comme les groupes de cellériers. Toutes, cependant, se recoupent en de nombreux points autour de questions économiques (formation, financement, aide juridique, organes de dialogue).

75. Pour plus de détails, V. Sœur GARret, op. cit., p. 37 et s.

76. Cette société détient aujourd'hui huit boutiques implantées dans les grandes villes de France, là où les communautés ne se situent généralement pas, et proches d'une clientèle 
d' «Artisanat monastique » achète donc les produits des congrégations, ce qui procure des revenus à ces dernières, et les revend à des particuliers ${ }^{77}$. L'ATC s'affirme ainsi comme un précieux intermédiaire qui a servi, et sert encore aujourd'hui, à organiser horizontalement comme verticalement la filière commerciale congréganiste en rapprochant à la fois les producteurs congréganistes entre eux pour qu'ils collaborent ou évitent de se concurrencer, et ces mêmes producteurs des consommateurs pour susciter des débouchés.

Or les conseils d'administration de l'ATC comme de sa société sont composés majoritairement de religieux issus des monastères qui approvisionnent les boutiques, lesquelles sont souvent tenues par des bénévoles. Une association épaulée par une société, toutes deux organisant un réseau de vente verticalement et horizontalement, un conseil d'administration tenu en majorité par les producteurs utilisant le réseau, des bénévoles pour vendre: c'est beaucoup au regard des conditions objectives de concurrence. Néanmoins, l'ATC n'a jamais été inquiétée jusqu'à présent, car l'éventualité d'une atteinte sensible à la concurrence n'a jamais été rapportée, même s'il existe une indéniable volonté de ne pas se concurrencer entre entreprises congréganistes vendant sur le marché ${ }^{78}$.

En contribuant largement à organiser une filière commerciale pour les entreprises congréganistes, l'ATC a, en effet, davantage multiplié l'offre congréganiste qu'elle ne l'a soustraite aux conditions d'une saine concurrence. Or les règles de concurrence ont bien pour fin de permettre une offre la plus large possible sur le marché au prix le plus attrayant pour le consommateur. C'est ce que permet l'ATC sans rompre non plus avec la logique du juste prix $^{79}$. En ceci, elle s'inscrit dans le contexte reconnu bénéfique tant par le législateur que la jurisprudence, de certains accords verticaux et horizontaux dès lors que ceux-ci présentent un bilan économique positif ${ }^{80}$. En outre, il

nombreuse. En outre, d'autres associations ou sociétés commerciales ont pu être constituées en lien direct avec l'ATC mais en toute indépendance juridique. C'est le cas de l'association «Les Amis des cloîtres» à Marseille créée le 5 févr. 2001. L'objet de cette association reste cependant identique à celui de l'ATC et, en cas de dissolution, les statuts prévoient la dévolution du patrimoine à l'ATC.

77. Auparavant, les produits étaient seulement mis en dépôt dans les boutiques, Sœur GARRET, op. cit., p. 43.

78. Ceci est conforme aux vœux de Pie XII, repris dans l'instruction Inter Praclara, V. supra note 48 .

79. La dimension du juste prix est également rappelée par l'instruction Inter Praclara, précit., $n^{\circ} 27 \& 2$.

80. C. com., art. L. 420-4; A. et G. DeCOCQ, Droit de la concurrence interne et communautaire, Paris, LGDJ, 6e éd. 2014, p. 296, nº 258 et s.; M. Pedamon et H. Kenfack, Droit commercial, 
y a peu de chances qu'en l'espèce, cet accord ayant donné naissance à l'ATC aboutisse à excéder les seuils ${ }^{81}$ au-delà desquels il n'est plus présumé avoir un effet positif sur la concurrence.

Enfin, il est important de tenir compte de l'esprit qui anime une telle coopération. Dans une entente classique, la coopération résulte seulement de la convergence d'intérêts particuliers dans un but commun qui peut être provisoire ou ne porter que sur un point précis de l'activité déployée sur le marché. Au contraire, la coopération développée par les institutions congréganistes repose sur des liens d'amitié qui dépassent le contexte économique, car ils ne sont pas mus par le seul espoir d'un profit égoïste. La considération du profit est même mineure au regard des rapports qui se sont créés au fil de la coopération mise en œuvre depuis l'après-guerre entre institutions congréganistes. Certaines vont jusqu'à confier une partie de leur production à d'autres pour leur procurer des revenus ${ }^{82}$. Or les règles de concurrence n'ont pas vocation à régir ou empêcher les amitiés dans les rapports économiques, mais seulement la convergence d'intérêts individuels visant à organiser la concurrence pour que chacun en tire avantage ${ }^{83}$.

\subsubsection{L'ASSOCIATION MONASTIC}

Le problème des ententes se pose de la même façon concernant l'association et le label «Monastic». Constituée uniquement d'entreprises congréganistes, l'association Monastic n'octroie son label qu'à ces dernières ${ }^{84}$. Ce label identifie avec certitude l'origine monastique des produits et permet aux distributeurs spécialisés dans de tels produits de ne se fournir qu'auprès de producteurs labellisés. Nous pourrions donc penser qu'il s'agit là d'une entente horizontale entre producteurs pour fermer l'accès au marché des produits monastiques tout en organisant la distribution par une entente verticale entre l'association et les sociétés distributrices des produits. En réalité, il n'en est rien pour plusieurs raisons.

Paris, Dalloz, $4^{e}$ éd. 2015, p. 547, n 553 et s.; C. Nourissat et B. DE Claviere-Bonnamour, Droit de la concurrence, Libertés de circulation, Paris, Dalloz, $4^{e}$ éd. 2013, p. 293, nº 323.

81. V. M.-A. Frison-Roche et M.-S. PAYET, op. cit., $\mathrm{n}^{\circ} 212$ (accords verticaux) et $\mathrm{n}^{\circ} 218$ (accords horizontaux).

82. Ainsi, les produits de l'abbaye bénédictine de Chantelle sont le résultat du travail de trois monastères. Outre l'aspect confraternel, la répartition du travail permet au principal producteur de ne pas investir de fortes sommes dans l'embauche de laïcs, l'achat de machines et la construction de bâtiments; l'équilibre entre prière et travail est maintenu et les coûts de production restent moindres.

83. En ce sens, V. M.-A. Frison-Roche et M.-S. PAYet, op. cit., n 215, p. 196.

84. V. J. Couard, op. cit., $\mathrm{n}^{\circ} 495$ et $\mathrm{s}$. 
Tout d'abord, le label ne concurrence pas nécessairement d'autres marques collectives ayant la même finalité protectrice; il s'y surajoute s'il en existe, et l'association n'exige pas de protection exclusive par son label pour en accorder l'usage.

Le label n'a pas non plus un objet anticoncurrentiel touchant à la fixation des prix ou aux conditions de vente. S'il n'est accordé que pour certains produits fabriqués selon certaines exigences attestant de leur origine monastique, c'est uniquement parce que son objet est de protéger ses détenteurs et d'identifier leurs produits pour plus de transparence sur le marché. Il est donc fédérateur tout en n'empêchant pas que d'autres produits revendiquent une origine monastique.

L'association comme le label ont ainsi pour but, non pas d'augmenter le pouvoir de marché de leurs créateurs et titulaires - ce qui aurait un caractère anticoncurrentiel - mais de réduire les coûts de mise sur le marché en organisant une protection commune contre le parasitisme économique de concurrents usurpant l'image monastique. De ce point de vue, l'association n'oblige pas davantage à adhérer systématiquement à des prestations annexes comme la participation à un guide ou un catalogue, sans que ses membres puissent par ailleurs faire de publicité de façon autonome en dehors de ce que décide l'association ${ }^{85}$.

Enfin, il ressort des éléments précités que le label n'a pas d'effet anticoncurrentiel, car il contribue à maintenir une offre plurielle sur le marché en protégeant les producteurs qui en sont titulaires, et à informer le consommateur sur la qualité et l'origine des produits.

Il faut ajouter à ces arguments de fond que le législateur français a admis, par un décret d'exemption de 1996, que les producteurs du domaine agricole bénéficiant de signes de qualité - ce que sont les labels - ne fussent pas sanctionnés par les règles sur les ententes ${ }^{86}$. Si cela concerne donc toutes les entreprises congréganistes membres de syndicats d'appellation, ou utilisant des labels biologiques, il est également possible d'y voir une volonté plus large du législateur d'étendre cette catégorie d'exemption à tout accord portant sur la vente au détail de produits alimentaires ou artisanaux bénéficiant d'un signe de qualité. Rappelons à cet égard que l'avis du Conseil de la concurrence de 2006 sur la filière du commerce équitable en France aborde

85. Sur de telles pratiques qui, si elles existent, portent atteinte à la concurrence, rappr. Cons. conc., déc. $\mathrm{n}^{\circ}$ 06-D-06, 17 mars 2006, relative à des pratiques mises en ceuvre dans le secteur de l'hébergement touristique en gîtes ruraux et en chambres d'hôtes.

86. D. $\mathrm{n}^{\circ}$ 96-499, 7 juin 1996. 
le problème du label en ce sens ${ }^{87}$ et n'hésite pas à évoquer l'éventualité d'une mesure d'exemption ${ }^{88}$.

Enfin, ces formes d'exemptions ne doivent pas faire oublier que les petites et moyennes entreprises (PME) échappent normalement, par des effets de seuil, aux dispositions sanctionnant les ententes tant en droit européen ${ }^{89}$ qu'en droit interne ${ }^{90}$, car les accords dont elles conviennent sont considérés comme n'affectant ni, par nature ${ }^{91}$, le commerce entre États membres, ni, sensiblement ${ }^{92}$, la concurrence sur le marché. Il semble que l'immense majorité des entreprises congréganistes entre dans ce cas de figure, parmi lesquelles toutes celles qui sont membres de l'association Monastic.

C'est dire en définitive qu'il y a peu de chances pour que les réseaux associatifs congréganistes, notamment ceux de l'ATC et de Monastic, soient aujourd'hui qualifiés d'ententes tant en raison de leurs buts que de leurs effets positifs et de leur taille sur le marché.

Plus fondamentalement, nous constatons ainsi que le droit de la concurrence pose lui-même des restrictions suffisamment importantes aux principes qu'il énonce pour permettre aux institutions congréganistes de constituer des réseaux indispensables à la préservation de la finalité religieuse de leur

87. Cons. conc., avis n 06-A-07, 22 mars 2006, pts 12 et s.; 14 et s.; 64 et s.; 72 et s.; à rappr. Cons. conc., déc. ${ }^{\circ}$ 05-D-22, 18 mai 2005, relative à des pratiques mises en ouvre par l'association "Agriculture et Tourisme en Dordogne-Périgord», spéc. pts 22-23: "Une démarche collective de qualité comme, par exemple, la constitution d'un label de qualité ou encore la constitution d'un système d'identification professionnelle [...] constitue une entente entre les entreprises qui adhèrent à cette démarche, mais elle ne peut a priori, être considérée comme un comportement anticoncurrentiel lorsqu'elle tend à l'amélioration de la qualité des produits et des services vendus aux consommateurs finaux. »; "Une telle entente serait, en revanche, anti-concurrentielle si les critères d'octroi d'un label dont la détention est indispensable pour exercer une activité n'étaient pas suffisamment objectifs et clairs et se prêtaient à une application discriminatoire, permettant ainsi d'évincer des concurrents du marché concerné par le label, par des moyens autres que ceux fondés sur les mérites des entreprises ou encore si la charte régissant le label contenait des clauses de prix imposés ou des restrictions territoriales absolues. »

88. Cons. conc., avis n ${ }^{\circ}$ 06-A-07, 22 mars 2006, précit., pts 90 et s.

89. TFUE, art. $101 \S 3$.

90. C. com., art. L. 420-4 II ; le texte se réfère à une exemption par décret pour chaque accord ou catégorie d'accord, renforçant ainsi les dispositions du droit européen.

91. Il doit pour cela s'agir d'entreprises indépendantes employant moins de 250 personnes et réalisant un chiffre d'affaires annuel inférieur ou égal à 50 millions d'euros, ou dont le total du bilan annuel n'excède pas 43 millions d'euros: Règl. (UE) nº 651/2014 de la Commission, annexe I, art. 1.

92. En général, tant que l'entente ne conduit pas les entreprises impliquées à détenir réellement ou potentiellement $10 \%$ des parts de marché. 
activité économique. Le droit des ententes n'apparaît donc pas incompatible avec un fait religieux économique à certains égards irréductible.

\subsection{LA POSSIBLE EXONÉRATION DE SANCTION POUR PERCEPTION D'UNE AIDE D'ÉTAT}

Reste la question de la compatibilité des aides d'État que peuvent percevoir certaines communautés religieuses avec les règles du marché intérieur de l'Union ${ }^{93}$.

Une récente affaire permet d'apporter quelque éclairage à ce sujet sans pour autant donner de réponse certaine. En l'espèce, une congrégation catholique espagnole avait obtenu un permis de construire pour la rénovation et l'extension d'un bâtiment d'une école située sur un terrain lui appartenant dans une commune près de Madrid. Ce bâtiment, une salle de 450 places assises, était destiné à accueillir des réunions, des cours, des conférences, etc. Dans un premier temps, comme tout contribuable, la congrégation s'acquitta d'un impôt de 23730,41 euros sur les constructions, les installations et les ouvrages, l'ICIO, lequel impôt a été introduit dans l'ordre juridique espagnol par l'arrêté du 5 juin 2001.

Toutefois, en vertu d'un concordat conclu en 1979 avec le Saint-Siège, le Royaume d'Espagne accorde à l'Église catholique différentes exonérations fiscales. La congrégation a donc demandé le remboursement de l'impôt payé sur la base de l'article IV, paragraphe 1, sous B), de l'accord de 1979. Or par décision du 6 novembre 2013, l'administration fiscale de la commune a rejeté cette demande, faisant valoir que l'exonération fiscale ne serait pas applicable en l'espèce, parce qu'il s'agirait d'une activité sans rapport avec les finalités religieuses de l'Église catholique.

Le litige a donc été soumis au tribunal administratif de Madrid (Juzgado Contencioso-Administrativo $\left.n^{\circ} 4\right)$ qui a formé une demande préjudicielle auprès de la Cour de justice de l'Union européenne. La question était de savoir si le fait, pour un État membre, d'exonérer une Église de certains impôts, et ce, également pour des activités qui n'ont pas une finalité strictement religieuse, est constitutif d'une aide d'État interdite par l'article 107, paragraphe 1, du Traité sur le fonctionnement de l'Union européenne (TFUE), car créant une distorsion sur le marché.

93. Sur les aides d'État en général et leur régime, V. A. et G. DecocQ, op. cit., nº 381 et s.; C. Nourissat et B. De Claviere-Bonnamour, op. cit., p. 365 et s. 
La réponse, toute en nuance, a été apportée par la Cour de justice de l'Union européenne dans un arrêt du 27 juin $2017^{94}$ après avoir estimé que la demande de décision préjudicielle était recevable. Ce n'était d'ailleurs pas la première fois que les instances européennes étaient sollicitées sur ce sujet des aides d'État concernant des entités à caractère religieux ${ }^{95}$.

Sur le fond, la Cour répond qu'une exonération fiscale, telle que celle en cause, dont bénéficie une congrégation appartenant à l'Église catholique pour des ouvrages réalisés dans un immeuble destiné à l'exercice d'activités dépourvues de finalité strictement religieuse, est susceptible de relever de l'interdiction énoncée à l'article 107, paragraphe 1 du TFUE si, et dans la mesure où, ces activités sont économiques, ce qu'il incombe à la juridiction de renvoi de vérifier ${ }^{96}$.

Pour savoir si l'exonération de certains impôts dont bénéficie une communauté religieuse est susceptible de constituer une aide d'État prohibée, la Cour procède en quatre temps ${ }^{97}$.

En premier lieu, elle recherche classiquement si la congrégation est susceptible d'être qualifiée d' «entreprise », au sens de l'article 107, paragraphe 1 du TFUE.

En deuxième lieu, elle recherche si l'exonération fiscale en cause au principal tend à accorder un avantage économique sélectif à la congrégation.

En troisième lieu, elle recherche si cette mesure constitue une intervention de l'État espagnol ou est permise au moyen de ressources de cet État membre.

Enfin, en quatrième lieu, elle recherche si ladite exonération est susceptible d'affecter les échanges entre les États membres et de fausser ou menacer de fausser la concurrence au sein du marché intérieur.

\subsubsection{SAVOIR SI LA CONGRÉGATION EST UNE ENTREPRISE}

S'agissant en premier lieu du point de savoir si la congrégation est susceptible d'être qualifiée d'entreprise, la Cour rappelle que la notion d'entreprise comprend, dans le contexte du droit de l'Union de la concurrence, toute entité

94. CJUE, 27 juin 2017, aff. C-74/16, Congregación de Escuelas Pías Provincia Betania c/ Ayuntamiento de Getafe: comm. de presse du même jour; L'actu concurrence, n 65/2017, obs. A. Ronzano.

95. Rappr. TPIUE, 15 sept. 2016, aff. T-219/13, Ferracci c/ Commission et T-220/13, Escuela elementarMaria Montessori c/ Commission: Europe, nov. 2016, p. 32, note L. IDOT.

96. CJUE, 27 juin 2017, aff. C-74/16, précit., § 90.

97. Ibid., § 40 . 
exerçant une activité économique, indépendamment du statut juridique de cette entité et de son mode de financement ${ }^{98}$. Peu importe à cet égard qu'il s'agisse d'une entité privée ou publique ${ }^{99}$ ou que, comme en l'espèce, l'activité en cause soit exercée par une communauté religieuse, du moment qu'elle peut être qualifiée d'économique ${ }^{100}$. Il suffit que l'entité concernée offre des biens ou des services sur un marché donné ${ }^{101}$, donc soit en concurrence avec d'autres opérateurs qui poursuivent un but lucratif ${ }^{102}$. Dès lors constituent des services susceptibles d'être qualifiés d' «activités économiques» les prestations fournies normalement contre rémunération, celle-ci constituant la contrepartie économique de la prestation en cause ${ }^{103}$.

À ce stade, la Cour opère alors une distinction entre les activités d'enseignement privé et les activités d'enseignement public financé en règle générale par le budget public et non par les élèves ou leurs parents, pour lesquelles l'État n'entend pas s'engager dans des activités rémunérées mais accomplit sa mission dans les domaines social, culturel et éducatif envers sa population ${ }^{104}$. Or dès lors qu'il n'est pas exclu qu'un même établissement puisse exercer plusieurs activités, à la fois économiques et non économiques, la Cour insiste sur le fait que cette diversification oblige l'établissement d'enseignement à tenir une comptabilité séparée en ce qui concerne les différents financements reçus, de manière à exclure tout risque de subvention «croisée» de ses activités économiques au moyen de fonds publics dont elle bénéficie au titre de ses activités non économiques ${ }^{105}$.

En l'espèce, la Cour observe que la congrégation exerce trois types d'activité au sein de l'école concernée: des activités strictement religieuses, un enseignement subventionné par l'État espagnol et un enseignement «libre ${ }^{106}$ » sans concours financier de l'État et cantonné à des enseignements préscolaire, extrascolaire et post-obligatoire. Par ailleurs, la Cour relève que la congrégation enseignante fournit des services complémentaires de restauration et de transport à ses élèves ${ }^{107}$. Elle tire de ces constatations que seules les activités

\author{
98. Ibid., \& 41. \\ 99. Ibid., \& 42. \\ 100. Ibid., § 43 . \\ 101. Ibid., \& 45 . \\ 102. Ibid., \& 46 . \\ 103. Ibid., \& 47. \\ 104. Ibid., §§ 48-50. \\ 105. Ibid., \& 51 . \\ 106. Nous dirions en France «hors contrat». \\ 107. CJUE, 27 juin 2017, aff. C-74/16, précit., § 52.
}


d'enseignement non subventionnées par l'État espagnol semblent avoir un caractère économique, puisqu'elles sont financées pour l'essentiel au moyen de participations financières privées aux frais scolaires ${ }^{108}$.

Partant, elle indique que, en pratique, c'est au juge national de déterminer si et dans quelle mesure les locaux en question sont affectés, à tout le moins en partie, à de telles activités économiques ${ }^{109}$. Concrètement, le tribunal administratif de Madrid, juridiction de renvoi, devra déterminer si la salle de conférences de l'école est dédiée à un usage relevant exclusivement de l'une ou de l'autre de ces activités d'enseignement, ou d'un usage mixte ${ }^{110}$.

À cette fin, la Cour propose une grille d'analyse: si l'usage en était réservé aux seules activités d'enseignement subventionnées par l'État espagnol, l'exonération fiscale en cause au principal ne relèvera pas de l'interdiction des aides d'État ${ }^{111}$; si, en revanche, l'usage de cette salle de conférences était dédié uniquement aux activités d'enseignement dites «libres», l'exonération d'ICIO serait susceptible de relever de cette interdiction ${ }^{112}$; enfin, en cas d'usage mixte, l'exonération fiscale serait également susceptible de relever de ladite interdiction, car la salle en question serait, partiellement mais malgré tout, affectée à une activité économique ${ }^{113}$.

Néanmoins, si la juridiction de renvoi parvenait à une telle conclusion, encore faudrait-il, pour que l'exonération de l'ICIO soit qualifiée d'«aide d'État», au sens de l'article 107, paragraphe 1 du TFUE, que les autres conditions posées par le texte soient remplies.

\subsubsection{SAVOIR SI LA CONGRÉGATION DISPOSE D'UN AVANTAGE ÉCONOMIQUE SÉLECTIF}

La deuxième condition tient à l'existence d'un avantage économique sélectif au profit de la congrégation ${ }^{114}$. Aux yeux de la Cour, il faudrait pour que cette condition soit remplie, d'abord, que l'ICIO constitue un impôt normalement dû par tous les contribuables qui à la fois effectuent des travaux de construction ou de rénovation et répondent aux critères retenus par le texte, puis ensuite que l'exonération totale et permanente de cet impôt dont

108. Ibid., §§ 57-58.

109. Ibid., \& 54 .

110. Ibid., \& 59 .

111. Ibid., § 60 .

112. Ibid., \& 61 .

113. Ibid., \& 62 .

114. Ibid., \& 73. 
bénéficient les institutions catholiques en vertu de l'arrêté du 5 juin 2001 ne constitue pas une mesure générale applicable indistinctement à tous les opérateurs économiques, mais bien une mesure a priori sélective. Or, selon la Cour, non seulement cela semble être le cas en pratique ${ }^{115}$ mais, en plus, il ne ressort d'aucun élément du dossier que l'exonération fiscale prévue par l'arrêté du 5 juin 2001 résulterait directement des principes fondateurs ou directeurs du système fiscal du Royaume d'Espagne et qu'elle serait nécessaire au fonctionnement ainsi qu'à l'efficacité de ce système ${ }^{116}$.

\subsubsection{SAVOIR SI L'AVANTAGE CONFÉRÉ À LA CONGRÉGATION REPOSE OU PÈSE SUR L'EMPLOI DE FONDS PUBLICS}

En outre, la Cour relève en l'espèce que l'exonération fiscale en cause, qui induit la suppression d'une charge qui devrait normalement grever le budget de la congrégation, a pour corollaire une diminution à due concurrence des recettes de la municipalité et, partant, paraît remplir la condition relative à une intervention de l'État au moyen de ses ressources ${ }^{117}$.

\subsubsection{SAVOIR SI L'AVANTAGE CONFÉRÉ À LA CONGRÉGATION CRÉE UNE DISTORSION DE CONCURRENCE}

Enfin, la dernière condition consiste à savoir quelle est l'incidence de l'avantage économique sur les échanges entre les États membres et sur la concurrence, pour voir s'il n'y a pas de distorsion sur le marché. À cet égard, la Cour rappelle les quasi-présomptions qui s'attachent à ces deux critères, puisqu'il suffit que l'aide accordée par un État membre renforce la position de certaines entreprises par rapport à celle d'autres entreprises concurrentes dans les échanges entre les États membres ${ }^{118}$ et qu'elle vise à libérer une entreprise des coûts qu'elle aurait normalement dû supporter dans le cadre de sa gestion courante ou de ses activités normales ${ }^{119}$ pour que l'une et l'autre de ces conditions soient en principe remplies.

Or en l'espèce, la Cour observe que l'exonération litigieuse pourrait rendre plus attrayante la fourniture des services d'enseignement de la congrégation par rapport à celle des services d'établissements également actifs sur le même

115. Ibid., $\$ \S 68-71$.

116. Ibid., \& 72 .

117. Ibid., $\S \S 74-76$.

118. Ibid., \& 79 .

119. Ibid., \& 80 . 
marché ${ }^{120}$. Il suffira seulement à la juridiction de renvoi de s'assurer que les mesures en cause ne relèvent pas des aides de minimis ${ }^{121}$.

C'est dire que les faveurs fiscales dont peuvent bénéficier les congrégations, si elles se justifient au regard de l'utilité sociale des activités menées par la communauté ${ }^{122}$, sont également susceptibles de constituer des aides d'État porteuses de distorsion de concurrence et, à ce titre, peuvent être remises en cause par l'administration fiscale elle-même.

Si les congrégations ayant une activité économique sont donc bien des entreprises au sens du droit de la concurrence, leur identité propre ne justifie de régime dérogatoire à celui des autres entreprises sur le marché que dans la mesure où l'exercice de la liberté religieuse et des finalités qui s'y attachent en termes de valeurs humaines demeure primordial: entre Dieu et l'Argent, il faut choisir ${ }^{123}$.

120. Ibid., \& 81 .

121. Ibid., § 82, sur les aides de minimis, V. A. et G. DeCocQ, op. cit. En l'espèce, se posait aussi la question de savoir si l'exonération en cause ne devait pas être considérée comme une aide existante dès lors que l'accord de 1979 entre l'Espagne et le Saint-Siège était antérieur à l'adhésion de cet État membre à la Communauté économique européenne en 1985. À cet égard, la Cour rappelle utilement que l'ICIO n'a été introduit qu'après cette adhésion. Dans ces conditions, si la juridiction de renvoi devait constater l'existence d'une aide d'État au bénéfice de la congrégation, il ne pourrait s'agir que d'une aide nouvelle, au sens de l'article $108 \S 3$ du TFUE.

122. V. en ce sens, J. Couard, op. cit., $\mathrm{n}^{\circ} 769$ et s.

123. Lc 16,13: «Nul serviteur ne peut servir deux maîtres [...], Dieu et l'argent. » 